Boise State University

ScholarWorks

$1-1-2015$

\title{
Characteristics of Child Sexual Assault Within a Child Advocacy Center Client Population
}

\author{
Faye M. Carlson \\ Boise State University \\ Jane Grassley \\ Boise State University \\ Janet Reis \\ Boise State University \\ Kelley Davis \\ St. Luke's Regional Medical Center
}


This is an author-produced, peer-reviewed version of this article. The final, definitive version of this document can be found online at Journal of Forensic Nursing, published by Lippincott, Williams \& Wilkins. Copyright restrictions may apply. doi: 10.1097/ JFN.0000000000000063

Characteristics of Child Sexual Assault Within a Child Advocacy Center Client Population

Faye M. Carlson MS, FNc, RN., Assistant Professor

Boise State University School of Nursing

Boise, Idaho

Jane Grassley, $\mathrm{PhD}, \mathrm{RN}, \mathrm{BBCLC}$, Professor

Boise State University School of Nursing

Boise, Idaho

Janet Reis, $\mathrm{PhD}$

Research Professor

College of Health Sciences, Boise State University

Kelley Davis, RN, BS

Correspondence

Faye M. Carlson MS, FNc, Boise State University School of Nursing,

1910 University Drive, Boise, Idaho, 83725-1840

E-mail: fayecarlson@boisestate.edu 


\title{
Characteristics of Child Sexual Abuse in a Child Advocacy Center
}

\begin{abstract}
This descriptive study summarized data from a Child Advocacy Center (CAC) to illustrate how such information might be used to profile the scope and character of child sexual abuse (CSA) at the community level. This detailed information is not available from national or state data but is needed to understand the circumstances of the children receiving services and the type of care they may need. Variables included victim demographics, type of sexual abuse and relationship to the perpetrator, and the person to whom the victim was most likely to disclose their sexual assault. A total sample of 841 cases reports was reviewed. Chi square tests were used to determine if there were statistically significant associations between the age groupings, type of abuse and the perpetrator according to age grouping. Those children most often seen at this CAC were female (73\%), white (67\%), and living with their mothers, with both parents, or with parent and stepparent (80\%). The incidence of CSA increased for girls across age groups. However, boys aged 6 to 10 years comprised the greatest percentage of the males (56\%) who experienced CSA. For all three age groups, over half of the perpetrators were identified as relatives. Most children ( $85 \%$ ) experienced high impact sexual abuse behaviors of fondling, penetration, or some combination thereof. Children most often disclosed CSA to their mothers. Understanding patterns of CSA at the local level provides guidance beyond national and state data to forensic nurses regarding child and family needs within their communities.
\end{abstract}




\section{Introduction}

Sexual violence against children remains a major global health concern. The World Health Organization [WHO](2014) reported that $20 \%$ of women and $5 \%$ to $10 \%$ of men experience sexual abuse during childhood. Within the United States (U.S.), the Centers for Disease Control and Prevention (CDC, 2013) estimated that in $2011,9 \%$ of the 681,000 victims of childhood maltreatment experienced sexual abuse. The National Children's Alliance (2014) reported that of the 294, 781 children seen in 2012 at a Child Advocacy Center (CAC), $62 \%$ were victims of sexual abuse. Child sexual abuse (CSA) involves children, aged 0 to 17 years old, in any contact or non-contact sexual act by an adult, regardless of whether or not force or coercion were involved. CSA also can involve two children where there is an age or power differential (Townsend \& Rheingold, 2013, p. 11). CSA as per the WHO (2003) includes sexual activity "for which the child is not developmentally prepared and cannot give consent, or that violates the laws or social taboos of society" (p. 76).

Although the sexual abuse of children continues as a significant health concern globally and in the United States, the ability to engage the public in addressing CSA is limited by the need for reliable estimates of its prevalence and scope (Barth, Bermetz, Trelle, \& Tonia, 2013; Townsend \& Rheingold, 2013). To address this limitation on a global level, Barth et al. (2013) conducted a meta-analysis of 55 studies from 24 countries to determine the current global prevalence of CSA. From their analysis, they reported a prevalence estimate of 8 to $31 \%$ for girls and 3 to $17 \%$ for boys worldwide. Within the U. S., the National Survey of Children's Exposure to Violence II (NatSCEV 11), which involved a telephone survey of 4503 children, aged 0 to 17 years old, estimated current trends for childhood exposure to a spectrum of violence including CSA. The researchers reported that almost $6 \%$ of the total sample experienced a 
sexual victimization and $2.2 \%$ experienced a sexual assault such as a rape or contact sex offense by adults or peers during the previous year. Further analysis of gender and age revealed a higher rate among 14 to 17 year old girls; $22.8 \%$ experienced a sexual victimization and $10.7 \%$ a sexual assault. This rate was lower for boys of the same age (10.3\% and $1.9 \%$. respectively). For this age group, $17.4 \%$ of girls and $4.2 \%$ of boys experienced a sexual assault during childhood (Finkelhor, Shattuck, Turner, \& Hamby, 2014).

This same research team conducted a study to estimate the likelihood of exposure to sexual abuse and assault by age 17 using a pooled sample of 708 17-year-olds, 781 15-year-olds, and 804 16-year-olds, which was drawn from three previous studies (Finkelhor, Turner, Ormrod, \& Hamby, 2005; Finkelhor, Turner, Ormrod, \& Hamby, 2009; Finkelhor et al., 2014). They found that the lifetime rate rose for girls from $16.8 \%$ for 15 year olds to $26.6 \%$ for 17 year olds. The rate was much lower for boys, rising from $4.3 \%$ for 15 years to $5.1 \%$ for 17 year olds. For this group of children, adult perpetrators were exclusively responsible for $11.2 \%$ of the CSA experienced by females and $1.9 \%$ for males; over half of the CSA was at the hands of juvenile perpetrators (Finkelhor et al., 2014). These two studies appear to support the importance of differentiating age and gender when analyzing CSA rates.

Understanding global and national CSA trends is important for establishing policy to address CSA as a population health problem. However, forensic nurses and their colleagues need information related to CSA characteristics within their communities in order to effectively advocate for and respond to the needs of child victims and their families (Murphy, Potter, Stapleton, Wiesen-Martin, \& Pierce-Weeks, 2010). Child Advocacy Centers offer a potential source of data for investigating the scope and characteristics of CSA in a local community. The 750 CACs located across the U. S. use inter-professional teams to provide a safe, child-focused 
environment where abused children and their families receive coordinated care in a single, childfriendly environment (Cross et al., 2008; Horner, 2008; Walsh, Cross, Jones, Simone, \& Kolko, 2007). They collect data such as the type of CSA experienced by the child victim, demographic characteristics of victims and perpetrator, and to whom the child disclosed the abuse.

CAC data have been used in studies of treatment and forensic issues. For example, Gonzalez (2013) used data from a local CAC to investigate the relationship of family and child demographic factors to victim completion of treatment for CSA or physical abuse. Edinburgh, Pape-Blabolil, Harpin, and Saewyc (2014) collected chart data from a hospital-based CAC to describe the contextual events, abuse experiences, and disclosure processes of adolescent victims of multiple perpetrator rape. Walsh, Jones, and Swicicki (2014) conducted a study to explore using case tracking data from a single CAC to investigate the process of criminal justice processing of adult perpetrators. They found that their study results helped the CAC study site set benchmarks around tracking criminal justice processing for the community.

These completed studies illustrate how data from CAC's can inform their community about the scope and nature of CSA taking place. The present study was undertaken at a local CAC to explore the characteristics of CSA within the community it serves with a particular focus on children's age and gender group differences. 'The purpose of this report is to summarize data extracted from this CAC to illustrate the type of information that might be collected to better inform local efforts to confront CSA. The information presented goes beyond the national and state level statistics available.

\section{Methods}

\section{Data Collection}


Data collection began after the study was approved by the researchers' university intuitional ethics review board (IRB). The researchers also received permission to conduct the study from a CAC located in the Northwest region of the U. S. Data were collected using CAC charts where findings were recorded of interviews with children and their parent(s) or guardians who sought services at the CAC for either physical or sexual abuse, or suspected physical or sexual abuse were recorded. To determine what services the family might need, interviews were conducted by a social worker using ComerHouse's Forensic interview protocol Rapport, Anatomy Identification, Touch Inquiry, Abuse Scenario, and Closure (RATAC) model (Anderson et al, 2010).

The RATAC is a semi-structured, non directive protocol for interviewing alleged victims of child sexual abuse. Using this protocol, the social worker began with a question to the child as to why they were at the Center today. If the children did not volunteer information on their own they were asked specific things about clothing (on/off, pulled up/down, how it got off/down), body positions of child and offender, if they were told/threatened not to tell, if abuse happened one time of more than one time, locations of abuse (e.g. whose house or what room), location of others, and how other people found out what happened. Depending on the act, the child was asked about erections and ejaculations and other touching of private parts. At the conclusion of the interview, the child was encouraged to tell someone right away is anyone ever touched their private parts. The forensic interview with the child was recorded and viewed by law enforcement personnel during the interview. With the assistance of age appropriate anatomical drawings and the occasional use of anatomical dolls, detailed information about the character of the sexual abuse was obtained. Additionally, the child's parent(s) or guardian(s) were interviewed by a social worker. 
Data were collected from the CAC charts by two undergraduate research assistants (RAs) under the supervision of the senior nursing faculty researcher. They used a data collection tool with 52 variables of interest developed by the research team from the relevant literature related to CSA and from data available in the CAC charts (Douglas \& Finkelhor, 2005; Finkelhor et al., 2005; Finkelhor et al., 2009). At the time of the study, the charts were all paper based and kept in a secured record room and in secured file cabinets at the CAC. The student RAs were introduced to the project and data collection protocol by the nursing faculty researcher and the $\mathrm{CAC}$ administrator. They were given supervised access to the record room and completed their abstraction while in the room. Data were collected between September 2010 and June 2012. Verification of accuracy of coding was done by the senior researcher directing the study with review of $5 \%$ randomly selected records. Very few errors in abstraction were found ( $1 \%$ of all entries checked). At the conclusion of the data collection period, the Microsoft Excel spreadsheet data were transferred to a thumb drive and imported into SPSS for analysis by one of the RAs.

During the time frame that the records were abstracted, there were 841 forensic interviews completed with children who had been maltreated or experienced maltreatment combined with physical and/or sexual abuse. Five were duplicate cases of children brought to the $\mathrm{CAC}$ twice during the study time period; these cases were excluded from the analysis. After excluding cases that did not involve sexual abuse, the sample consisted of 519 victims of CSA between the ages of 2 and 17 years old. Eighteen year olds were excluded because they are defined as adults in the state where the study was conducted.

\section{Data Coding and Analysis}


After reviewing relevant literature, the victims were grouped into three age categories: 2 to 5 years old, 6 to 10 years old, and 11 to 17 years old (Finkelhor et al., 2009; Murphy et al., 2010). Following the categorization scheme of Martin and Silverstone (2013) the cases were initially grouped into four CSA categories or types. Type one CSA involves non-contact such as being forced to watch a person masturbate or having pictures taken. Type two encompasses genital touching (e g. specific types include fondling genitals, oral genital contact, fondling anus fondling offender). Types three and four include behaviors related to penetration, either attempted (type 3) or actual (type 4), of an object, finger, or penis. 'These four original categories were further classified to combine non contact and genital touching; non contact, genital touching, and penetration; non contact and penetration; and genital touching and penetration (See Iable 2).

The head of household data was only available for 292 of the total 519 sexual abuse victims. In the original data collection template, "head of household" was an open text field. These data were re-coded into eight categories that included mother, father, parents, parent and step-parent, parent with significant other, grandparent(s), foster parent(s), and other. If the child was living with the mother and some other person, such as a grandmother or other relative, or the mother's friend, this was re-coded to "mother" as head of household. The "other" category included guardian, aunt, uncle, cousin, unknown, and state facility.

The original data collection template had 39 categories under "Disclosed to whom", (See Table 3). These categories were combined into seven final categories. For example, "mother" included biological/adoptive mother, stepmother, or foster mother. The category "professional" included teacher, principal, counselor, social worker/PSR worker, youth/church leader and/or police/resource officer. The category "other" included family members other than the mother, 
friends, boyfriends, girlfriends, victim disclosure through writing, a witness of the CSA, and unknown/unclear.

\section{Results}

\section{Demographics}

Those children most often seen at this CAC were female (73\%), and the majority were white $(67 \%) ; 20 \%$ were of two or more races and $13 \%$ were Hispanic or Latino (See Table 1). The frequency of CSA increased for girls across age groups from $18 \%$ ( 2 to 5 year olds) to $38 \%$ ( 6 to 10 year olds) to $45 \%$ (11 to 17 year olds) of total females. For all males, frequency of abuse differed by age with more boys aged 6 to 10 years (56\%) abused compared to 2 to 5 year olds $(21 \%)$ and 11 to 17 year olds (23\%). The rate of CSA in the 11 to 17 year old group was five times greater for females $(84 \%)$ than that for males $(16 \%)$.

Of the children for whom head of household was reported, $80 \%$ lived with their mother (35\%), both parents (25\%), or parent and stepparent $(17 \%)$. The number of children residing with a parent and stepparent increased as the age of the children increased. The category of father as the head of household had the least number of cases. There was no statistically significant association between child's age grouping and race or head of household. Age category was significantly associated with gender (chi square $=21.17, \mathrm{p}<.000$ ).

\section{Type of Abuse by Perpetrator and Age}

Table 2 describes the coded type of abuse by age category and the two most frequently reported perpetrators (e. g. relatives or acquaintances). The majority ( $85 \%$ ) experienced high impact sexual abuse behaviors of fondling, penetration or some combination thereof. The number of children in the 2 to 5 year old group with no events reported for non contact abuse, genital touching and penetration, and non contact and penetration precluded analysis of the 
relationship between age, type of abuse, and perpetrator. For the older age groups there were too many cells with expected counts of less than 5 to perform reliable tests of the relationship between age, perpetrator, and type of abuse. Analysis of the overall relationship between the older age groups and perpetrator and between the older age groups and type of abuse showed a significant association between age group and type of abuse (chi square $=31.38, p<.000$ ), but none between age group and type of perpetrator.

\section{Disclosure}

Of the 421 children who disclosed their sexual abuse, almost half disclosed to their mothers (See Table 3). There was a statistically significant association between the age categories and to whom the child disclosed (chi square $=35.67, \mathrm{p}<.000$ ). As the age of the child increased, the number of children disclosing to professionals or to others such as family members or friends increased.

\section{Discussion}

Girls in this study experienced CSA at a higher rate than boys, a finding consistent with the literature (Barth et al., 2013; Douglas \& Finkelhor, 2005; Finkelhor et al., 2013; Finkelhor et al.., 2014; Martin \& Silverstone, 2013). The literature suggests that rate of CSA is similar for girls and boys in the younger age groups, but changes as children enter adolescence. The rates of CSA for girls increase, while decreasing for boys (Finkelhor et al., 2014; Martin \& Silverstone, 2013), which is congruent with this study's findings. Among the males in this study, $56 \%$ were 6 to 10 years old. This differs from the findings reported by Finkelhor et al. (2009) where 2.1\% of 6 to 9 year old males experienced sexual victimization and $1.4 \%$ experienced sexual assault.

The majority of children in this study ( $85 \%$ ) most often experienced fondling and/or penetration, which are considered higher-impact CSA because of their long-term health 
consequences. Victims of this type of abuse are at risk for increased rates of psychiatric illnesses such as anxiety, eating disorders, depression and posttraumatic stress disorder as well as problems with cognitive and social development (Chen et al., 2010; Martin \& Silverstone, 2013; Pérez-Fuentes et al., 2013).

One of the challenges of addressing CSA involves disclosure of the abuse. Children rarely disclose the abuse soon after an incident, making it difficult to investigate. Children in this study most often disclosed to their mothers, a finding supported by the literature (Bick, Zajac, Ralston, \& Smith, 2014). Young children may not have the language or the cognitive developmental skills to understand or describe what happened to them (Fontes \& Plummer, 2010). Boys may be reluctant to disclose the abuse because of fears related to their sexual identity (Perez-Fuentes, 2013; Lippert, Cross, Jones, \& Walsh, 2011).

The identity of the perpetrator influences disclosure. The vast majority of children in this study knew their perpetrators, which is widely documented in the literature (Douglas \& Finkelhor, 2005; Murphy et al., 2010). Studies estimate that $50 \%$ of perpetrators are acquaintances; the estimate for relatives is more disparate (14\% to $47 \%$ ) (Douglas \& Finkelhor, 2005; Murphy et al., 2010). In this study, relatives comprised the majority of perpetrators in all age groups, with $62 \%$ of the 2 to 5 year old group experiencing CSA by a relative compared to $33 \%$ by an acquaintance. A similar finding was reported by Murphy et al. (2010); for children younger than 6 years old; $44 \%$ of perpetrators were relatives compared to $38 \%$ acquaintances.

\section{Limitations}

This study has several limitations. Because data were collected retrospectively from charts that were for clinical, rather than research purposes, the preciseness of the data is affected by the accuracy of the charting. 'The geographical setting of the study may limit generalizability 
of the findings to other regions. Self report presents another limitation because the victims and /or family members may choose to limit the information they provide to the CAC interviewer. Missing data, particularly related to demographics, is also a limitation. Nonetheless, the data assembled here provide additional insight into the experiences of children within one community and how information from a CAC might be used to further understand a child's risk for abuse.

\section{Implications for Clinical Forensic Nursing Practice}

The findings of this study fill a gap in the literature noted by Martin and Silverstone (2013) of the need to determine relationships between age group, gender, and type of CSA within a specific geographical location. Awareness of these relationships can assist forensic nurses as they screen potential victims of CSA. Kendall-Tackett, Williams, and Finkelhor (1993) argued that age and gender be differentiated in studies of CSA because a child's developmental stage and gender impact his/her interpretation and response to the abuse. Although over 20 years old, their recommendations from their systematic literature review of the impact of CSA are still relevant for health professionals.

The severity of the CSA experienced by the children brought to the local CAC provides guidance to forensic nurses as to the scope of need for treatment and the potential for long term negative impact. Further, mothers tended to be the person to whom the child first disclosed the abuse. Although this is a well documented phenomenon in the literature on CSA, documentation at a local level is a reminder of the importance of verifying within the context of a defined community. Forensic nurses' strategic offering of support to mothers so that they can offer support to their child is an essential aspect of a child's healing process. However, nurses should bear in mind that the mother's response to the disclosure influences the support she can offer to 
her child, particularly if she has been abused herself by the same perpetrator (Bick et al., 2014;

Godbout. Briere, Sabourin, \& Lussier, 2013).

A final implication of this study for forensic nursing is the potential benefits of partnerships between nurse researchers and the CAC interprofessional team. In this study the nurse researchers were able to assist the CAC staff in developing a usable database for describing and analyzing the characteristics of the population they serve.

\section{Conclusions}

This descriptive study demonstrates the importance of age and gender in understanding the dynamics of child sexual abuse. In addition local Children's Advocacy Centers have potential to be reliable sources of data regarding the characteristics of child sexual abuse in a particular community. This information can be used to help communities develop resources for the identification and treatment of victims, an important priority given the long-term health consequences of CSA. 


\section{References}

Anderson, J., Ellefson, J., Lashley, J., Lukas Miller, A., Olinger, S., Russell, A., Stauffer, J., \& Weigman, J. (2010). The Cornerhouse forensic interview protocol: RATAC. Thomas $M$. Cooley Journal of Practical and Clinical Law, 12, 193-331.

Barth, J., Bermetz, E., Helm, E., Trelle, S., \& Tonia, T. (2013). The current prevalence of child sexual abuse world - wide: A systematic review and meta-analysis. International Journal of Public Healih Online, 58(3), 1-15, doi:10.1007/s00038-012-0426-1

Bick, J., Zajac, K., Ralston, M. E., \& Smith, D. (2014). Convergence and divergence in reports of maternal support following childhood sexual abuse: prevalence and associations with youth psychosocial adjustment. Child Abuse and Neglect, 38(3), 479-487. doi:10.1016/j.chiabu.2013.11.010.Epub2014 Jan20.

Centers of Disease Control and Prevention [CDC]. (2013). Child maltreatment: Facts at a glance. Retrieved from: www.cdc.gov/violenceprevention/pdf/cm-data-sheet--2013.pdf.

Chandler, N. (2000) Best practices for establishing a children's advocacy center $\left(3^{\text {rd }}\right.$ ed.).Washington, DC. National Children's Alliance.

Chen,L., Murad, M.,H., Paras, M., Colbenson, K., Sattler, A., Goranson, N., ...Zirakzadeh, A. (2010). Mayo Clinic Proceedings. Jul 2010; 85(7), 618-629. doi: $10.4065 / \mathrm{mcp} .2009 .0583$

Cross, T. P., Jones, L. M., Walsh, W. A., Simone, M., Kolko, D. J., Szczepanski, J., ... Magnuson, S. (2008, August). Evaluating children's advocacy centers' response to child sexual abuse. Juvenile Jistice Bulletin. U. S. Department of Justice. Retrieved from www.ojp.usdoj.gov 
This is an author-produced, peer-reviewed version of this article. The final, definitive version of this document can be found online at Journal of Forensic Nursing, published by Lippincott, Williams \& Wilkins. Copyright restrictions may apply. doi: 10.1097/ JFN.0000000000000063

Douglas, E., M., \& Finkelhor, D. (2005). Childhood sexual abuse fact sheet. Crimes Against Children Research Center. Retrieved from www.unh.edu/ccrcl

Edinburgh, L., Pape-Blabolil, J., Harpin, S. B., \& Saewyc, E. (2014). Multiple perpetrator rape among girls evaluated at a hospital-based child advocacy center: Seven years of reviewed cases. Child Abuse \& Neglect, 38(9), 1540-1551.

Finkelhor, D., Ormrod, R., Turner, H. A., \& Hamby, S. L. (2005). The victimization of children and youth: A comprehensive, national survey. Child Maltreatment, 10(1), 5-25. doi: $10.1177 / 107755950427128$

Finkelhor, D., Shattuck, M. A., Turner, H. A., \& Hamby, S .L. (2014). The lifetime prevalence of child sexual abuse and sexual assault assessed in late adolescence. Journal of Child and Adolescent Health, 55, 329-333.

Finkelhor, D., Turner, H. A., Ormrod, R., \& Hamby, S. L. (2009). Violence, abuse, and crime exposure in a national sample of children and youth. Pediatrics,124(5), 14111423. doi:10.1542/peds.2009-0467

Fontes, L., \& Plummer, C. (2010). Cultural and disclosure issues: Cultural issues in disclosures of child sexual abuse. Journal of Child Sexual Abuse, 19, 491-518.

Godbout, N., Briere, J., Sabourin, S., \& Lussier, Y. (2014). Child sexual abuse and subsequent relational and personal functioning: The role of parental support. Child Abuse $\&$ Neglect, 38, 317-325.

Gonzalez, J. E. (2013). Influence of family and victim demographic factors on treatment completion for children exposed to abuse and family violence. University Of Central Florida Undergraduate Research Journal, (6)2, 94-101. 
Horner, G. (2008). Child advocacy centers: Providing support to primary care providers. Journal of Pediatric Health Care, 22(1), 35-39.

Kendall-Tackett, K. A., Williams, L. M., \& Finkelhor, D. (1993). Impact of sexual abuse on children: A review and synthesis of recent empirical studies. Psychological Bulletin, 113(1), 164-180. doi: 10.1037/0033-2909.113.1.164

Lippert, T., Cross,T., Jones, L., \& Walsh, W. (2011). Telling interviews about sexual abuse: Predictors of child disclosure at forensic interviews. Child Maltreatment, 14(1), 100-113. doi: $10.1177 / 1077559508318398$

Martin, E. \& Silverstone, P. (2013, July). How much child sexual abuse is "Below the Surface," and can we help adults identify it early? Frontiers in Psychiatry, 4(58). Retrieved from: http://www.ncbi.nlm.nih.gov/pmc/articles/PMC37I 1274.

Murphy, S.B., Potter, S.J., Stapleton, J.G., Wiesen-Martin, D. \& Pierce-Weeks, J. (2010). Findings from sexual assault nurse examiners (SANE): A case study of New Hampshire's pediatric SANE database. Journal of Forensic Nursing, 6, 163-169.

National Children's Alliance (NCA). (2013). NCA national statistics: Statistical report for 2013. Retrieved from www.nationalchildrensalliance.org/? $\mathrm{s}=36$.

Perez-Fuentes, G/, M. Olfson, M., L. Villegas, L., C. Morcillo, C., S. Wang, S., C. Blanco, C. (2013). Prevalence and correlates of child sexual abuse: A national study. Comprehensive Psychiatry, 54, 16-27. doi.org/10.1016/j.comppsych.2012.05.010

Townsend, C., \& Rheingold, A. A. (2013). Estimating a child sexual abuse prevalence rate for practitioners: A review of child sexual abuse prevalence studies. Charleston, SC: Darkness to Light. 
This is an author-produced, peer-reviewed version of this article. The final, definitive version of this document can be found online at Journal of Forensic Nursing, published by Lippincott, Williams \& Wilkins. Copyright restrictions may apply. doi: 10.1097/ JFN.0000000000000063

Walsh, W., Cross, T., Jones, L., Simone, M., \& Kolko, D. (2007). Which sexual abuse victims receive a forensic medical examination? The impact of children's advocacy centers. Child Abuse \& Neglect, 31, 1053-1068.

Walsh, W. A., Jones, L. M., \& Swiecicki, C. C. (2014). Using child advocacy center tracking data to examine criminal disposition times. Journal of Child Sexual Abuse, 23(2), 198-216. doi: $10.1080 / 10538712.2014 .868386$

World Health Organization. (WHO, 2003). Guidelines for medico-legal care for victims of sexual violence. gender and women's health, family and community health injuries and violence prevention, noncommunicable diseases and mental health. Retrieved from: http://whqlibdoc.who.int/publications/2004/924154628X.pdf.

World Health Organization. (WHO, 2014). Child maltreatment: Fact Sheet. Retrieved from http://www.who.int/mediacentre/factsheets/fs150/en/ 
This is an author-produced, peer-reviewed version of this article. The final, definitive version of this document can be found online at Journal of Forensic Nursing, published by Lippincott, Williams \& Wilkins. Copyright restrictions may apply. doi: 10.1097/ JFN.0000000000000063

\section{Table 1}

Socio-demographic Characteristics by Age

\begin{tabular}{lccc}
\hline & $2-5$ Year olds (\%) & 6 -10 Year olds (\%) & $11-17$ Year olds (\%) \\
Gender & $67(70)$ & $142(64)$ & $169(84)$ \\
Female & $29(30)$ & $79(36)$ & $33(16)$ \\
Male & & & \\
Race & $68(71)$ & $151(68)$ & $131(65)$ \\
White/Caucasian & $9(9)$ & $24(11)$ & $32(16)$ \\
Hispanic & $19(20)$ & $46(21)$ & $39(19)$ \\
Other & & & \\
Head of Household & $17(30)$ & $43(36)$ & $45(39)$ \\
Mother & $2(4)$ & $4(3)$ & $3(3)$ \\
Father & $21(38)$ & $35(29)$ & $20(17)$ \\
Both parents & $2(4)$ & $18(15)$ & $31(27)$ \\
Parent and stepparent & $7(13)$ & $4(3)$ & $5(4)$ \\
Parent and significant & & & $3(3)$ \\
other & $3(5)$ & $7(6)$ & $3(4)$ \\
Grandparent(s) & $1(2)$ & $7(6)$ & $5(4)$ \\
Foster parent(s) & $3(5)$ & $2(2)$ & \\
Other & & & \\
Missing 227 & & & \\
& & & \\
\hline
\end{tabular}


Table 2

Type of Abuse by Perpetrator and Child's Age ${ }^{a}$

\begin{tabular}{|c|c|c|c|c|c|c|}
\hline \multicolumn{7}{|c|}{ Years } \\
\hline & $\begin{array}{l}2-5 \\
\text { Relative }\end{array}$ & $\begin{array}{l}2-5 \\
\text { Acquaintance }\end{array}$ & $\begin{array}{l}6-10 \\
\text { Relative }\end{array}$ & $\begin{array}{l}6-10 \\
\text { Acquaintance }\end{array}$ & $\begin{array}{l}\text { 11-17 } \\
\text { Relative }\end{array}$ & $\begin{array}{l}11-17 \\
\text { Acquaintance }\end{array}$ \\
\hline Non Contact & $9(16)$ & $8(27)$ & $16(13)$ & $3(11)$ & $20(44)$ & $11(50)$ \\
\hline $\begin{array}{l}\text { Genital } \\
\text { Touching }\end{array}$ & $29(53)$ & $9(31)$ & $57(46)$ & $35(55)$ & $31(27)$ & $20(31)$ \\
\hline $\begin{array}{l}\text { Both Non } \\
\text { Contact And } \\
\text { Genital } \\
\text { Touching }\end{array}$ & $4(7)$ & $1(3)$ & $15(12)$ & $15(56)$ & $14(49)$ & $11(41)$ \\
\hline Penetration & $5(9)$ & $1(3)$ & $12(10)$ & $1(10)$ & $1(6)$ & $8(80)$ \\
\hline $\begin{array}{l}\text { Non Contact, } \\
\text { Genital } \\
\text { Touching } \\
\text { And } \\
\text { Penetration }\end{array}$ & $3(5)$ & 0 & $11(9)$ & $5(20)$ & $17(55)$ & $20(80)$ \\
\hline $\begin{array}{l}\text { Non Contact } \\
\text { And } \\
\text { Penetration }\end{array}$ & 0 & 0 & $3(2)$ & $1(8)$ & $4(57)$ & $12(92)$ \\
\hline $\begin{array}{l}\text { Genital } \\
\text { Touching } \\
\text { And } \\
\text { Penetration }\end{array}$ & $5(9)$ & $10(34)$ & $12(10)$ & $7(32)$ & $1(6)$ & $5(23)$ \\
\hline $\mathrm{N}$ & 55 & 29 & 122 & 68 & 96 & 87 \\
\hline
\end{tabular}


This is an author-produced, peer-reviewed version of this article. The final, definitive version of this document can be found online at Journal of Forensic Nursing, published by Lippincott, Williams \& Wilkins. Copyright restrictions may apply. doi: 10.1097/ JFN.0000000000000063

Table 3

Victim Disclosure by Age

\begin{tabular}{lccc}
\hline Person & $\begin{array}{c}2-5 \text { Year olds } \\
(\%)\end{array}$ & $\begin{array}{c}6-10 \text { Year olds } \\
(\%)\end{array}$ & $\begin{array}{c}11-17 \text { Year olds } \\
(\%)\end{array}$ \\
Mother & $\begin{array}{c}39(50) \\
\text { Mother and father }\end{array}$ & $80(46)$ & $61(36)$ \\
Father & $13(17)$ & $10(6)$ & $8(5)$ \\
Sibling(s) & $2(3)$ & $13(7)$ & $12(7)$ \\
Grandparent(s) & $5(6)$ & $6(3)$ & $13(8)$ \\
Professional & $1(1)$ & $15(9)$ & $4(2)$ \\
Other & $18(23)$ & $18(10)$ & $23(14)$ \\
& & $32(18)$ & $48(28)$ \\
\end{tabular}

